Article

\title{
A Novel Approach to Perform the Identification of Cross-Section Deformation Modes for Thin-Walled Structures in the Framework of a Higher Order Beam Theory
}

\author{
Lei Zhang ${ }^{1, * \mathbb{D}}$, Aimin $\mathrm{Ji}^{1, *}$ and Weidong $\mathrm{Zhu}^{2}{ }^{2}$ \\ 1 College of Mechanical and Electrical Engineering, Hohai University, Changzhou 213022, China \\ 2 Department of Mechanical Engineering, University of Maryland, Baltimore County, \\ Baltimore, MD 21250, USA; wzhu@umbc.edu \\ * Correspondence: leizhang@hhu.edu.cn (L.Z.); jam@ustc.edu (A.J.); \\ Tel.: +86-0516-8519-1840 (L.Z.); +86-0516-8519-1959 (A.J.)
}

Received: 3 November 2019; Accepted: 26 November 2019; Published: 29 November 2019

Featured Application: The novel approach, together with the resulting higher-order beam model, possess both efficiency and accuracy in capturing three-dimensional behaviours, which makes it an alternative to more sophisticated shell theories in dynamic analyses of thin-walled structures.

\begin{abstract}
This paper presents a novel approach to identify cross-section deformation modes for thin-walled structures by assembling preliminary deformation modes (PDM) considering their participation in free vibration modes. These PDM, defined over the cross-section through kinematic concepts, are integrated in the governing equations of a higher order model and then uncoupled in the form of generalized eigenvectors. The eigenvectors are deemed to inherit the attributes of structural behaviours and can serve as the basis to assemble PDM. Accordingly, a criterion was developed to handle the eigenvectors, pursuing (i) the clustering of PDM that participate in a same structural behaviour, (ii) the assignation of the corresponding weights that indicate their participation and (iii) the decomposition of an amplitude function when it is related to several structural behaviours. Moreover, a numbering system was proposed to hierarchically organize the deformation modes, which is conducive to a reduced higher order model. The main features of this approach are found in its ability to be performed in a more operational way and its nature to give deformation modes physical interpretation inherited from the dynamic behaviours. The versatility of the approach was validated through both numerical examples and comparisons with other theories.
\end{abstract}

Keywords: thin-walled structures; cross-section deformation modes; a higher order beam theory; generalized eigenvectors; relevance analysis

\section{Introduction}

Thin-walled beams are widely used as structural components in weight-sensitive industrial applications, largely due to the advantage of high strength to weight ratio. However, they are also prone to cross-section deformation due to "thin walls", which may cast significant influences on the mechanical performance [1]. Therefore, it is essential to consider the phenomenon in the design process. Classic beam theories fail to achieve this, since they preclude the detection of cross-section warping and distortion. To overcome this, many attempts have been made to develop new beam theories for thin-walled structures over the years. 
Among them, one normal strategy adopted is to reduce the three-dimensional (3D) elasticity formulation to a one-dimensional (1D) model. Such models are usually quite exact since no further assumptions are added. Typically, the Saint-Venant solution (SVS) has been the theoretical basis of several thin-walled beam models. For example, see El Fatmi [2] and Genoese et al. [3]. By adding enrichment terms to the Saint Venant solution, various non-Classic effects can be taken into account in beam models. Detailed implementations can be found in Naccache and Fatmi [4]. However, the derived solutions are always problem-dependent [5]. Variational asymptotic methods (VAM) represent another powerful tool, which allows the decomposition of a 3D elasticity problem into a 1D formulation [6]. Now, it has stepped toward the refined beam model with a transcendental accuracy [7]. However, the solution is in closed form for only a few cross-section geometries and materials. In order to deal with arbitrary cross-sections, the variational asymptotic beam sectional analysis (VABS) was developed based on VAM, exploiting the finite element method over the cross-section to solve the variational problem and deriving beam-like equations for higher order effects [8]. Related work can be found in studies by Harursampath et al. [9] and Jiang [10].

A recent contribution to refined beam theories is the Carrera unified formulation (CUF) models, where the order of the theory is optional and related to the convergence analysis. As a hierarchical formulation, it can reduce 3D problems to $2 \mathrm{D}$ or $1 \mathrm{D}$ by considering the definition of the displacement field through arbitrary expansions [11]. Over the past decade, CUF has been developed for the higher order analysis of thin-walled structures. Some valuable developments were reported by Pagani et al. [12] and Yan et al. [13]. It should be noted that it allows special improvements on the accuracy of representing higher order effects [14]. Moreover, CUF is not dependent of the structural problem and thus no extra assumptions should be made to represent a particular structural effect. Therefore, this formulation has been successfully adopted in the modelling of thin-walled beams with arbitrary cross-sections [15].

Some refined formulations have also been developed by considering the addition of representative deformation modes. The cross-section nonuniform warping defined by Vlasov [16] was the first higher order mode introduced to refine the displacement field. From then on, various warping functions have been formulated. The work reported in Capdevielle et al. [17] and Yoon et al. [18] are typical examples. Some approaches have focused on the use of shear correction factors to enhance classic beam theories. These shear correction factors provide a more appropriate shear distribution over the beam cross-section and thus accommodate the stress-free boundary conditions. Shear correlation factors can be defined in various ways, but are dependent on problem characteristics [19]. In the field of bridge engineering, some higher order deformation modes have been introduced to capture special structural behaviours or load conditions [20]. The analyses of the multi-cell distortion [21], torsional warping [22] and distortional warping [23] due to shear-lag effects in box girders are successful examples.

There are some existing beam theories exploring a general procedure to define deformation modes. The generalized beam theory (GBT) [24] considers the deformed configuration through a linear combination of deformation modes. These modes are derived with the cross-section analysis [25], which provides deformation modes being hierarchically organized. At present, GBT has been well-established as an efficient and versatile tool to perform first-order [26], buckling [27], vibration [28], post buckling [29] and dynamic analyses [30] of thin-walled structures in elasticity. Another procedure to define deformation modes was proposed by Vieira et al. [31]. It considers an enrichment of the displacement field on the cross-section through a set of interpolation functions defined over a mesh of the cross-section, and a set of uncoupled deformation modes representing higher order effects are derived based on the solution of a polynomial eigenvalue problem [32,33]. Thus, the resulting higher order model is capable to cope with the loss of accuracy inherent from the reduction of a 3D elasticity formulation to a 1D model [34].

The present paper focused on a systemic but simple procedure to identify deformation modes considering dynamic behaviours of thin-walled structures. The procedure was supposed to obtain an exhaustive set of deformation modes for a thin-walled cross-section and open to determine the 
hierarchy with the structural geometry and material parameters, as well as boundary conditions taken into account. To this end, three steps should be taken. First, preliminary deformation modes (PDM) were defined through kinematic assumptions and integrated in governing equations. They were uncoupled by solving the generalized eigenvalue problem associated with homogeneous governing equations. Second, PDM were assembled to generate final deformation modes. Within each eigenvector, the collinear amplitude functions can be viewed as a set of quantities describing the same structural behaviour. In this sense, the set of PDM can be assembled together to generate a final deformation mode. The key was to address the issues including the determination of PDM with collinear amplitude functions, the assignation of the weights indicating the participation and the decomposition of an amplitude function when related to several structural behaviours. Finally, the assembled deformation modes were hierarchically organized, and further employed to derive a reduced higher order beam model. In order to illustrate the application of the proposed approach, numerical examples were presented concerning representative cross-sections.

\section{A Higher Order Model}

A prismatic thin-walled structure was considered in formulating the higher order model. As shown in Figure 1, the cross-section was constituted by rectilinear walls, including both open and closed profiles. The global coordinate system $(x, y, z)$ is also shown. The local coordinate systems $(s, n, z)$ were adopted in each wall, where $s$ defines the wall mid-line and $n$ indicates the thickness $t$.

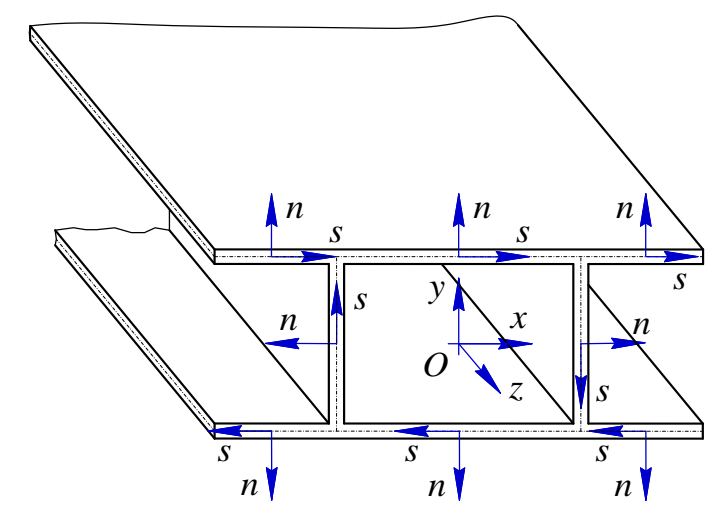

Figure 1. Global $(x, y, z)$ and local $(s, n, z)$ coordinate systems of the illustrative thin-walled structure.

\subsection{Displacement Field}

The displacement of an arbitrary point on the thin-walled structure was expressed with three components $U, V$ and $W$ in the local coordinate system. By considering the membrane and flexural behaviours, the 3D displacement field $\mathbf{D}=[U(s, n, z), V(s, n, z), W(s, n, z)]^{\mathrm{T}}$ can be expressed by

$$
U(s, n, z)=u(s, z)-n w, z(s, z), V(s, n, z)=v(s, z)-n w, s(s, z), W(s, n, z)=w(s, z)
$$

where (i) the subscript commas indicate differentiation with respect to the following variable, and (ii) the variables, $u, v$ and $w$, are the mid-plane displacement components along $z$, $s$ and $n$, respectively. By separating the variable dependencies on $s$ and $z$, these components were obtained with the linear combination of deformation modes (including $N_{1}$ out-of-plane and $N_{2}$ in-plane ones) as

$$
u(s, z)=\sum_{k=1}^{N_{1}} \phi_{k}(s) \chi_{k}(z), v(s, z)=\sum_{h=1}^{N_{2}} \psi_{h}(s) \chi_{N_{1}+h}(z), w(s, z)=\sum_{h=1}^{N_{2}} \omega_{h}(s) \chi_{N_{1}+h}(z)
$$

where (i) $k$ and $h$ are the numbers of out-of-plane and in-plane deformation modes, respectively; (ii) $\varphi$, $\psi, \omega$ are the deformation components of deformation modes along $z, s$ and $n$, respectively; and (iii) $\chi$ is 
the amplitude function varying along $z$. Notice that the shape functions are initially defined based on PDM and finally replaced by identified deformation modes to describe the displacement field.

Substitute Equation (2) into Equation (1), and the 3D displacement field can be rewritten in a 1D form by involving a transformation matrix $\mathbf{H}$ as

$$
\mathbf{D}(s, n, z)=\mathbf{H x}, \mathbf{H}=\left[\begin{array}{cc}
\boldsymbol{\varphi} & -n \boldsymbol{\omega} \frac{\partial}{\partial z} \\
0 & \boldsymbol{\psi}-n \boldsymbol{\omega}, s \\
0 & \boldsymbol{\omega}
\end{array}\right]
$$

where (i) the amplitude function vector $\mathbf{x}$ is spanned with amplitude functions $\chi$, and (ii) $\varphi, \psi$ and $\boldsymbol{\omega}$ are the shape function vectors consisting of $\varphi(s), \psi(s)$ and $\omega(s)$ in row matrices, respectively.

The strain and stress fields were obtained under the small displacement hypothesis. By applying the Kirchhoff formulation, the strain $\varepsilon=\left[\varepsilon_{z z}(s, n, z), \varepsilon_{s s}(s, n, z), \gamma_{s z}(s, n, z)\right]^{\mathrm{T}}$ and stress fields $\sigma=\left[\sigma_{z z}(s\right.$, $\left.n, z), \sigma_{s s}(s, n, z), \tau_{s z}(s, n, z)\right]^{\mathrm{T}}$ can be obtained as

$$
\begin{gathered}
\mathcal{\varepsilon}(s, n, z)=\mathbf{C D}, \mathbf{C}=\left[\begin{array}{ccc}
\frac{\partial}{\partial z} & 0 & 0 \\
0 & \frac{\partial}{\partial s} & 0 \\
\frac{\partial}{\partial s} & \frac{\partial}{\partial z} & 0
\end{array}\right] \\
\boldsymbol{\sigma}(s, n, z)=\mathbf{E} \varepsilon, \mathbf{E}=\left[\begin{array}{ccc}
\frac{E}{1-v^{2}} & \frac{E v}{1-v^{2}} & 0 \\
\frac{E v}{1-v^{2}} & \frac{E}{1-v^{2}} & 0 \\
0 & 0 & \frac{E}{2(1+v)}
\end{array}\right]
\end{gathered}
$$

where (i) $\mathbf{C}$ and $\mathbf{E}$ are the compatibility operator and the constitutive matrix, respectively, and (ii) $E$ and $v$ are the material Young's modulus and Poisson's ratio, respectively.

\subsection{Governing Equations}

The governing equations were obtained through Hamilton's principle, with the strain energy $U_{\text {st }}$, the kinetic energy $T_{\mathrm{kn}}$ and the potential energy $U_{\mathrm{pt}}$ involved. By definition, $U_{\mathrm{st}}$ and $T_{\mathrm{kn}}$ are given by

$$
\begin{gathered}
U_{\mathrm{st}}=\frac{1}{2} \iiint_{V} \varepsilon^{\mathrm{T}} \boldsymbol{\sigma} \mathrm{d} V \\
T_{\mathrm{kn}}=\frac{1}{2} \iiint_{V} \rho \frac{\partial \mathbf{D}^{\mathrm{T}}}{\partial t} \frac{\partial \mathbf{D}}{\partial t} \mathrm{~d} V
\end{gathered}
$$

where $V$ is the beam volume and $\rho$ is the material density. The beam is supposed to bear distributed loads $\mathbf{p}=[p, q, r]^{\mathrm{T}}$, where $p, q$ and $r$ represent the force densities along $z$, $s$ and $n$, respectively. Thus, $U_{\mathrm{pt}}$ can be given by

$$
U_{\mathrm{pt}}=-\int_{L} \int_{A} \mathbf{D}^{\mathrm{T}} \mathbf{p} \mathrm{d} A \mathrm{~d} z
$$

Here, Hamilton's principle yields the governing equations as

$$
\int_{L} \int_{A} \mathbf{H}^{\mathrm{T}} \mathbf{C}^{\mathrm{T}} \rho \mathbf{C H} \frac{\partial^{2} \mathbf{x}}{\partial t^{2}} \mathrm{~d} A \mathrm{~d} z+\int_{L} \int_{A} \mathbf{H}^{\mathrm{T}} \mathbf{C}^{\mathrm{T}} \mathbf{E C H} \mathbf{x} \mathrm{d} A \mathrm{~d} z-\int_{L} \int_{A} \mathbf{H}^{\mathrm{T}} \mathbf{p} \mathrm{d} A \mathrm{~d} z=0
$$

where $A$ and $L$ are the cross-section area and the beam length, respectively.

For the ease of computation, the governing equations are usually calculated with the finite element method. Here, quadratic Lagrange functions are suggested for the longitudinal interpolation in deriving the finite element. Details were described in a previous study [35]. 


\section{Identification of Deformation Modes}

This section presents the approach to perform the identification of cross-section deformation modes. In order to better exhibit the procedure, the main steps are illustrated by taking an example of the cross-section shown in Figure 1. Similar to GBT [36], PDM are defined on the basis of kinematic hypotheses and then assembled to generate final deformation modes.

\subsection{Preliminary Deformation Modes}

PDM are defined on a meshed cross-section. A prismatic cross-section can be viewed as a "network" of a minimum number of straight walls. The nodes on these walls were divided into three types: The key, the natural and the artificial ones. The walls were bound to cross-link at intersection nodes, and the two intersection nodes closest to the wall ends were referred to as key nodes, while the other ones were classified into natural nodes. The nodes located at wall free ends were considered natural nodes. A selective number of artificial nodes may also be introduced to divide long walls into segments, when the wall is notably long. The nodes were numbered around clockwise in the sequence of key nodes, natural nodes and artificial nodes. The result is shown in Figure 2, where a set of $N=$ 12 nodes, including $n_{\mathrm{d}}=4$ key nodes, $n_{\mathrm{f}}=4$ natural nodes and $n_{\mathrm{a}}=4$ artificial nodes, carried out the discretization of the cross-section. Figure $2 \mathrm{a}$ also shows the cross-section geometry, having a height of $h=0.6 \mathrm{~m}$, a width of $b=0.8 \mathrm{~m}$ and a flange width of $c=0.4 \mathrm{~m}$.

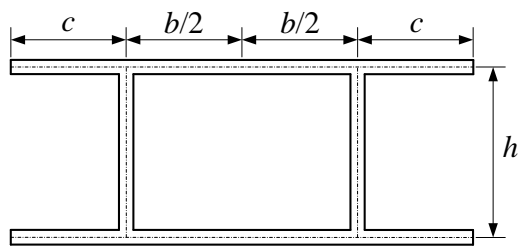

(a)

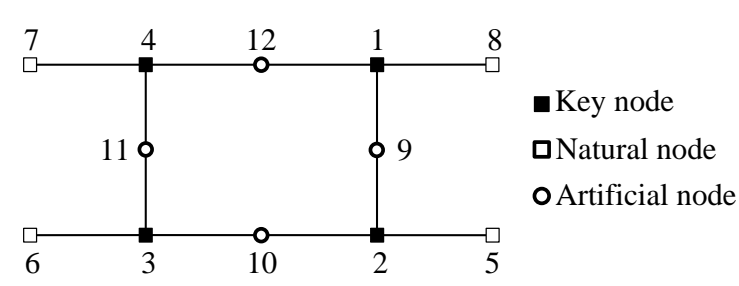

(b)

Figure 2. (a) Geometry of the illustrative cross-section and (b) the cross-section discretization.

The definition of PDM is related to the imposition of unit displacements on the discretization nodes. Here, four degrees of freedom at each node were considered, including three translations along three orthogonal axes and one rotation about the longitudinal axis. Thus, the discretization leads to a total of $4 \times N$ PDM. Note that different types of nodes were imposed different displacement boundaries in the procedure: For a key node $i$, the unit displacement was imposed on it and null displacements on all other key nodes; for a natural node $j$, the related deformation mode corresponded to the imposition of a unit displacement on it and null displacements on all key nodes and other natural nodes; and for an artificial node $k$, the related deformation modes were obtained by imposing a unit displacement on node $k$ and null displacements on all other nodes.

In order to be included in the higher order model, PDM should be mathematically described with shape functions about $s$. Here, the shape functions were defined through the interpolation of the imposed unit displacements. For convenience, these shape functions were decomposed into components being consistent with $z, s$ and $n$, respectively. The interpolation polynomials should satisfy the continuity condition along $s$. To ensure the efficiency, different interpolation polynomials were adopted for different shape functions. For example, a set of linear Lagrange functions was used to describe the axial deformation $\varphi_{k}(s)$ and tangential deformation $v_{k}(s)$, while the normal deformation $\omega_{k}(s)$ employs a set of cubic Hermite functions. For a better presentation, the resulted PDM of the illustrative cross-section were numbered from 1 to 48 and exhibited in a matrix form in Figure 3.

These PDM can be divided into three families: (i) The Dominant family, including those stemming from the imposition of translational unit displacements on key nodes; (ii) Integral family, composing those involving natural nodes; and (iii) Selective family, with all other ones included. This is conducive 
to the separation of classic modes and higher order modes. For example, the classic modes can only be assembled with members of the Dominant family (probably revised with the members of Integral family), while the Selective family is dedicated to generate higher order modes.

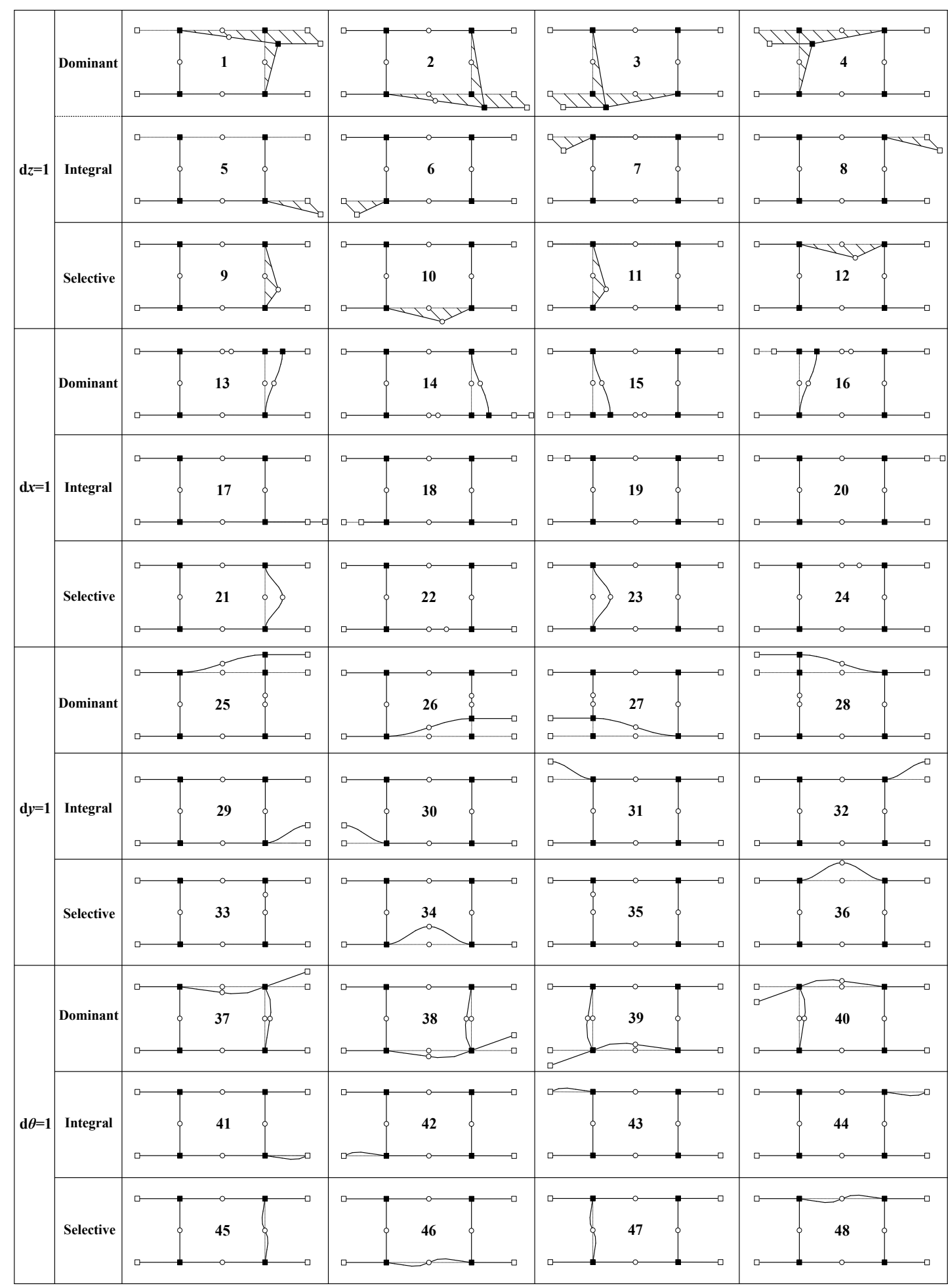

Figure 3. Preliminary deformation modes (PDM) of the illustrative cross-section stemming from the imposition of four-unit displacements on each of the 12 discretization nodes. 


\subsection{Cross-Section Deformation Modes}

The PDM shown in Figure 3 were derived purely through kinematic concepts and was not derived through structural interpretation. Thus, a procedure was developed to assemble PDM into three main families: (i) The classic modes, which are consistent with those of the Timoshenko theory; (ii) Primary modes, which are independent with Classic modes in certain vibration modes; and (iii) Secondary modes, which accompany Classic or Primary modes in vibration modes with relatively smaller participation. Among them, the priority is in a descending order in influencing the structural performance. This is just the basis to form a reduced thin-walled beam model: Classic and Primary modes are qualified to derive a higher order model being applicable for most cases, and a selective number of Secondary modes can be supplemented into the model in special cases for a higher accuracy.

The initial governing equations are a coupled system, and it is difficult to have a deep sight in the relations of PDM in time domain. However, the amplitude functions can be uncoupled by solving the eigenvalue problem related to the homogenous governing equations, namely

$$
\int_{L} \int_{A} \mathbf{H}^{\mathrm{T}} \mathbf{C}^{\mathrm{T}} \rho \mathbf{C H} \frac{\partial^{2} \mathbf{x}}{\partial t^{2}} \mathrm{~d} A \mathrm{~d} z+\int_{L} \int_{A} \mathbf{H}^{\mathrm{T}} \mathbf{C}^{\mathrm{T}} \mathbf{E} \mathbf{C H} \mathbf{x} \mathrm{d} A \mathrm{~d} z=0 .
$$

In the process, the finite element implementation adopts the quadratic Lagrange interpolation and leads to $\mathbf{x}=\mathbf{N X}$. As a result, the eigenvectors are derived and grouped in a matrix form $\mathbf{X}$ as

$$
\mathbf{X}=\left[\mathbf{x}^{(1)}, \mathbf{x}^{(2)}, \cdots, \mathbf{x}^{(k)}, \cdots, \mathbf{x}^{(N)}\right]
$$

where the superscript $(k)$ is the order of an eigenvector. Notice that $\mathbf{x}^{(k)}$ is grouped with $N$ amplitude functions as $\mathbf{x}^{(k)}=\left[\chi_{1}(z), \chi_{2}(z), \ldots, \chi_{N}(z)\right]^{\mathrm{T}}$, and the amplitude function $\chi_{i}(z)$ can be expressed in a discretization form as

$$
\chi_{i}=\left[\chi_{i}(1), \chi_{i}(2), \cdots, \chi_{i}\left(N_{\mathrm{e}}\right)\right]^{\mathrm{T}}
$$

where $N_{\mathrm{e}}$ is the number of interpolation nodes along $z$. In other words, an eigenvector $\mathbf{x}^{(k)}$ is actually spanned with $N$ amplitude function vectors $\chi_{i}{ }^{(k)}$ which further contain $N_{\mathrm{e}}$ node values. Thus, it can be rewritten in a matrix form as

$$
\mathbf{x}^{(k)}=\left[\mathbf{x}_{1}{ }^{(k)}, \mathbf{\chi}_{2}{ }^{(k)}, \cdots, \chi_{N}{ }^{(k)}\right] \in \mathbb{R}^{N_{\mathrm{e}} \times N}
$$

where each column represents the axial variation of an amplitude function. More precisely, the eigenvector $\mathbf{x}^{(k)}$ is now reformed as an "eigen matrix".

The eigen matrix is deemed to inherit the structural behaviours and possess the information to assemble PDM. Within each eigen matrix, the collinear amplitude functions can be viewed as the quantities describing the same structural behaviour. In this sense, the corresponding set of PDM can be assembled together considering their participation. Therefore, one has to address the issues including determining the collinear amplitude functions (DCAF) in an executable way, assigning the weights of the deformation modes (AWDM) based on their participation and decomposing an amplitude function into components (DAFC) when related to multiple structural behaviours.

\subsubsection{Basic Algorithms}

Here, some basic algorithms were employed to handle the eigen matrices. For each eigen matrix $\mathbf{x}^{(k)}$, one should check the participation $\rho_{i}$ of a PDM $i$ in advance, since not all of them are effective. The participation $\xi_{i}$ is defined as

$$
\xi_{i}=\frac{\left\|x_{i}{ }^{(k)}\right\|_{\infty}}{\max \left(\left\|x_{1}{ }^{(k)}\right\|_{\infty},\left\|\chi_{2}{ }^{(k)}\right\|_{\infty}, \cdots,\left\|\chi_{i}{ }^{(k)}\right\|_{\infty}, \cdots,\left\|\chi_{N}{ }^{(k)}\right\|_{\infty}\right)}
$$


where \|\|$_{\infty}$ is the infinite norm of a vector. In practice, the PDM with a participation lower than the threshold $\xi_{0}=0.001$ are ignored since it has little impact on the structural performance. The PDM with participations larger than 0.001 are referred to as effective amplitude function vectors (EAFV).

To address the first issue DCAF, the Pearson correlation coefficient was employed to distinguish the relationship between the two amplitude function vectors. The correlation coefficient $r_{i, j}$ between $\chi_{i}^{(k)}$ and $x_{j}^{(k)}$ is defined as

$$
r_{i, j}=\frac{\operatorname{cov}\left(x_{i}^{(k)}, \chi_{j}^{(k)}\right)}{\sqrt{\operatorname{var}\left[x_{i}^{(k)}\right] \operatorname{var}\left[x_{j}^{(k)}\right]}}
$$

where the symbols $\operatorname{cov}()$ and $\operatorname{var}[]$ are the covariance and the variance, respectively. A coefficient $r_{i, j}=1$ or -1 is the sign of collinear amplitude function vectors. Generally, PDM with a mutual correlation coefficient of 1 or -1 are deemed to be describe the same structural behaviour and can thus be assembled together to form a final deformation mode after AWDM.

For the second issue AWDM, the weight $\lambda_{i, j}$ of a PDM $j$ is assigned as

$$
\lambda_{i, j}=\frac{\left\|x_{j}{ }^{(k)}\right\|_{\infty}}{\left\|x_{i}^{(k)}\right\|_{\infty}}
$$

where $\chi_{i}^{(k)}$ is the amplitude function selected as the standard reference (the unit weight). Notice that the amplitude function vectors in Equation (16) should be collinear EAFV, and that the standard reference is usually confined to the one having the maximum participation.

The third issue DAFC confronts the situation that $\operatorname{PDM} j$ may participate in several structural behaviours, being characterized by correlation coefficients equal to neither 1 nor -1 . The situation can be further divided into two cases according to the type of deformation families involved.

In the first case, the amplitude function vectors of the Dominant family are collinear, but an amplitude function vector $\chi_{j}^{(k)}$ of other families should be decomposed into two components $\beta_{1}$ and $\beta_{2}$. Then, the two components can be obtained as

$$
\boldsymbol{\beta}_{1}=\frac{\operatorname{dot}\left(\chi_{j}^{(k)}, \chi_{i}^{(k)}\right)}{\operatorname{dot}\left(\chi_{i}^{(k)}, \chi_{i}^{(k)}\right)} x_{i}^{(k)}, \beta_{2}=\chi_{j}^{(k)}-\beta_{1}
$$

where $\operatorname{dot}()$ is the inner product of $\chi_{i}{ }^{(k)}$ and $\chi_{j}{ }^{(k)}$, and $\chi_{i}{ }^{(k)}$, as the standard reference, should only participate in this structural behaviour. Thus, the derived component $\beta_{1}$ is bound to be collinear with $\chi_{i}^{(k)}$, while the residual component $\beta_{2}$ is linearly independent. The procedure is referred to as DAFC 1 (the first case of DAFC).

In the second case, the amplitude function vectors of the Dominant family are not collinear, with mutual correlation coefficients close to 1 or -1 . It indicates two structural behaviours, I and II (usually two Classic modes or a Classic mode and a Primary mode) involved, and another procedure is needed to decompose both the two amplitude function vectors $\chi_{i}^{(k)}$ and $\chi_{j}^{(k)}$ into two components. Naturally, the two vectors can be expressed as

$$
\boldsymbol{\chi}_{i}^{(k)}=\boldsymbol{\beta}_{i, \mathrm{I}}+\boldsymbol{\beta}_{i, \mathrm{II}}, \boldsymbol{x}_{j}^{(k)}=\boldsymbol{\beta}_{j, \mathrm{I}}+\boldsymbol{\beta}_{j, \mathrm{II}}
$$

where the symbol $\beta$ indicates amplitude function components and the subscripts I and II denote different structural behaviours. Furthermore, the two components belonging to a same structural behaviour should be collinear, reading

$$
\boldsymbol{\beta}_{i, \mathrm{I}}=\mu_{1} \boldsymbol{\beta}_{j, \mathrm{I}}, \boldsymbol{\beta}_{i, \mathrm{II}}=\mu_{2} \boldsymbol{\beta}_{j, \mathrm{II}}
$$


where $\mu_{1}$ and $\mu_{2}$ are the undetermined coefficients. In fact, the proportion of amplitude functions for a Classic mode is predictable. The point is to identify the type of the classic mode involved. This problem was discussed in a previous paper [37], where the Classic modes were recognized through the signs of benchmark points. Thus, Equation (18) and Equation (19) yield

$$
\left(\mu_{2}-\mu_{1}\right) \beta_{i, I I}=\chi_{j}^{(k)}-\mu_{1} \chi_{i}^{(k)}
$$

which means the component $\beta_{i, \mathrm{II}}$ is collinear with the linear combination $\boldsymbol{\chi}_{j}^{(k)}-\mu_{1} \boldsymbol{\chi}_{i}{ }^{(k)}$. Therefore, an orthogonalization procedure can be used to further decompose all the amplitude function components. Based on this, the components can be obtained as

$$
\begin{aligned}
& \boldsymbol{\beta}_{i, \mathrm{I}}=\chi_{i}^{(k)}-\frac{\operatorname{dot}\left(\chi_{j}{ }^{(k)}-\mu_{1} \chi_{i}{ }^{(k)}, \chi_{i}{ }^{(k)}\right)}{\operatorname{dot}\left(\chi_{j}{ }^{(k)}-\mu_{1} \chi_{i}{ }^{(k)}, \chi_{j}{ }^{(k)}-\mu_{1} \chi_{i}\left({ }^{(k)}\right)\right.}\left(\chi_{j}{ }^{(k)}-\mu_{1} \chi_{i}{ }^{(k)}\right), \boldsymbol{\beta}_{i, \mathrm{II}}=\chi_{i}{ }^{(k)}-\boldsymbol{\beta}_{i, \mathrm{I}},
\end{aligned}
$$

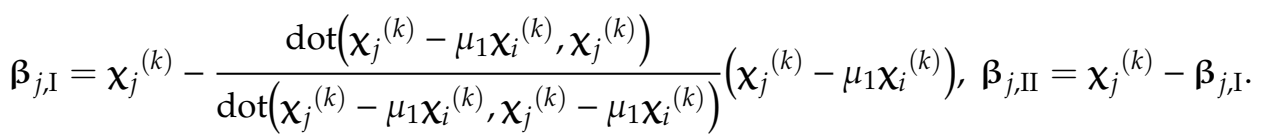

The process is referred to as DAFC 2 (the second case of DAFC).

In the process of DAFC, a possible problem is how to decide whether a residual component should be further studied or just ignored. Hence, the residual participation $\xi_{\mathrm{r}}$ is defined as the indicator. When the residual component $\beta_{2}$ obtained from DAFC satisfies the condition

$$
\xi_{\mathrm{r}}=\frac{\left\|\boldsymbol{\beta}_{2}\right\|_{\infty}}{\max \left(\left\|x_{1}{ }^{(k)}\right\|_{\infty},\left\|\chi_{2}{ }^{(k)}\right\|_{\infty}, \cdots,\left\|\chi_{i}{ }^{(k)}\right\|_{\infty}, \cdots,\left\|\chi_{N}{ }^{(k)}\right\|_{\infty}\right)} \leq \xi_{0}
$$

then the component $\beta_{2}$ can be ignored. Otherwise, it should be assembled with other PDM.

Finally, the shape function $\eta(s)$ of an identified deformation mode can be obtained as

$$
\eta(s)=\sum_{j} \frac{r_{i, j}}{\left|r_{i, j}\right|} \frac{\operatorname{dot}\left(\boldsymbol{x}_{i}^{(k)}, \boldsymbol{\chi}_{j}^{(k)}\right)}{\operatorname{dot}\left(\boldsymbol{x}_{i}^{(k)}, \boldsymbol{\chi}_{i}^{\left({ }^{(k)}\right)}\right.} \theta_{j}(s)
$$

where $\theta_{j}(s)$ is the shape function of a $\operatorname{PDM} j, \chi_{i}{ }^{(k)}$ is the amplitude function vector selected as the standard reference and all the amplitude function vectors related are collinear EAFV.

One should bear in mind that the algorithms above can handle two vectors each time. In practice, a certain number of amplitude functions are processed in pairs according to a well-planned sequence. Details are discussed in the following section.

\subsubsection{Concrete Implementation}

For a better implementation, the amplitude function vectors of each eigen matrix were divided into two groups (representing the out-of-plane and in-plane PDM, respectively) and separately handled using the basic algorithms. Besides, the order of the eigen matrix and the type of PDM were considered in planning the sequence: The eigen matrix of lower order has priority than those of higher order, and within each eigen matrix, amplitude functions are dealt with in the sequence of Dominant family, Integral family and Selective family. For a better grasp of the procedure, a cantilevered thin-walled structure with an illustrative cross-section and a beam length of $L=6.4 \mathrm{~m}$ was modelled to illustrate the concrete implementation.

The procedure first focused on the out-of-plane PDM of the first eigen matrix. By checking the participation, it was discovered that only 10 of the 12 out-of-plane PDM had participations exceeding 0.001 (as show in Table 1). The corresponding amplitude function vectors were recognized as EAFV for further analysis. Next, the correlation coefficients were calculated, and the results support that $\chi_{1}{ }^{(1)}$ 
can be selected as the standard reference since it has the biggest participation among the amplitude functions of the Dominant family. As shown in the third column of Table 1, the correlation coefficients $\left|\gamma_{1, i}\right|=1.0000(i=1,2,3,4)$ indicate that amplitude functions of these PDM were collinear. According to the signs of the benchmark points, it can be determined that the related classic mode was the bending about $x$ axis, since $\gamma_{1,2}=-1, \gamma_{1,3}=-1$ and $\gamma_{1,4}=1$. Thus, they were assembled to derive the first classic mode by employing the weights (the fourth column of Table 1) calculated with AWDM. The result is shown in Figure 4a.

Table 1. The participations, correlation coefficients and weights of the out-of-plane preliminary deformation modes for the first eigenvector of the illustrative thin-walled structure.

\begin{tabular}{|c|c|c|c|c|c|c|c|}
\hline $\operatorname{PDM} i$ & Participation $\xi_{i}$ & $\begin{array}{c}\text { Correlation } \\
\text { Coefficient } r_{1, j}\end{array}$ & Weight $\lambda_{1, j}$ & $\begin{array}{c}\text { Residual } \\
\text { Participation } \xi_{i}\end{array}$ & $\begin{array}{c}\text { Correlation } \\
\text { Coefficient } r_{5, j}\end{array}$ & Weight $\lambda_{5, j}$ & $\begin{array}{c}\text { Residual } \\
\text { Participation } \xi_{i}\end{array}$ \\
\hline 1 & 0.1085 & 1 & 1 & 0 & 1 & / & 0 \\
\hline 2 & 0.1085 & -1.000 & -1.000 & 0 & / & / & 0 \\
\hline 3 & 0.1085 & -1.000 & -1.000 & 0 & / & / & 0 \\
\hline 4 & 0.1085 & 1.000 & 1.000 & 0 & / & / & 0 \\
\hline 5 & 0.0022 & / & / & 0.0022 & 1 & 1 & 0 \\
\hline 6 & 0.0022 & I & I & 0.0022 & 1.000 & 1.000 & 0 \\
\hline 7 & 0.0022 & / & / & 0.0022 & -1.000 & -1.000 & 0 \\
\hline 8 & 0.0022 & I & / & 0.0022 & -1.000 & -1.000 & 0 \\
\hline 11 & 0.0017 & I & / & 0.0017 & 0.5874 & 0.7181 & 0.0004 \\
\hline 12 & 0.0017 & / & l & 0.0017 & -0.5874 & -0.7181 & 0.0004 \\
\hline
\end{tabular}
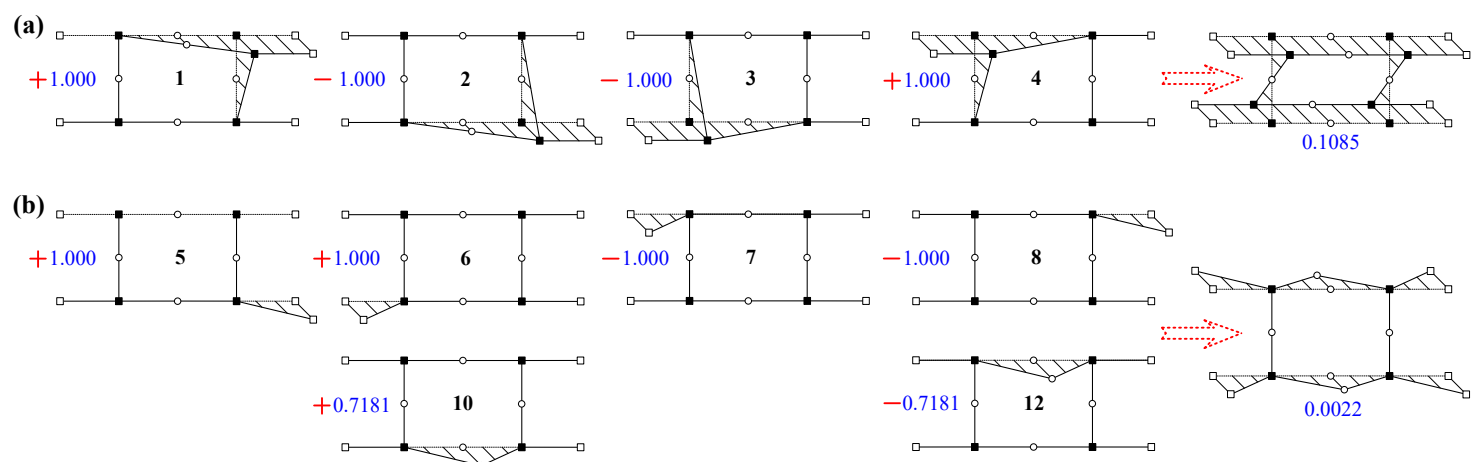

Figure 4. The assembling of the out-of-plane preliminary deformation modes by handling the first eigenvector of the illustrative thin-walled structure: (a) a classic mode and (b) a warping mode.

Then, the implementation goes on with the other six EAFV. Among them, $\chi_{5}{ }^{(1)}$ was selected as the standard reference since it had the biggest participation (see the fifth column of Table 1). The calculation of correlation coefficients indicated that not all of them were collinear, which gave top priority to the Integral family. Thus, DAFC 1 was applicable for the further decomposition. Then, $\chi_{10}{ }^{(1)}$ and $\chi_{12}{ }^{(1)}$ were decomposed into two components, respectively. The first component was collinear with $\chi_{5}{ }^{(1)}$, and the other component should be ignored since the residual participation was below 0.001 . The weights of the first components were derived and shown in the seventh column. Consequently, there was no EAFV left. The assembling of the new deformation mode is shown in Figure $4 \mathrm{~b}$.

The assembling of the in-plane PDM needed to handle more data since more amplitude functions were involved. It was essential to determine the EAFV by checking the participations. Then, EAFV were classified into teams where the absolute correlation coefficients were bigger than $\gamma_{1}=0.9$. Among each team, the standard reference was selected in the light of the biggest participation. Then, the correlation coefficients were calculated to carry out DCAF, which states $\gamma_{25,26}=\gamma_{25,27}=\gamma_{25,28}=1$, which indicates the Classic mode of translation along the $y$ axis, according to the signs of benchmark points. The weights were obtained through AWDM. After screening out these dominant modes, the left 24 EAFV were divided into two teams: $\chi_{29}{ }^{(1)} \sim \chi_{32}{ }^{(1)}, \chi_{34}{ }^{(1)}$, and $\chi_{36}{ }^{(1)} \sim \chi_{44}{ }^{(1)}$ as the first team, and $\chi_{13}{ }^{(1)} \sim \chi_{20}{ }^{(1)}, \chi_{33}{ }^{(1)}$ and $\chi_{35}{ }^{(1)}$ as the other team. Besides, one should note that some amplitude function vectors are exactly the same in mathematics. This property contributes to further reducing the 
number of amplitude function vectors to be processed. Table 2 shows the results, where the retained EAFV are separated into two teams. Within the first team, $\chi_{29}{ }^{(1)}$ was selected as the standard reference, and the others were decomposed into components using DAFC 1. As shown in the fifth column, the residual participations of $\chi_{37}{ }^{(1)}$ and $\chi_{39}{ }^{(1)}$ still exceeded 0.001 . In this case, they were absorbed into the second team for further analysis. Within the second team, $\chi_{13}{ }^{(1)}$ was selected as the standard reference. Subsequently, the other amplitude function vectors were decomposed and assigned weights using DAFC 1 and AWDM, respectively. The assembling result is shown in Figure 5.

Table 2. The participations, correlation coefficients and weights of the in-plane preliminary deformation modes for the first eigenvector of the illustrative thin-walled structure.

\begin{tabular}{|c|c|c|c|c|c|c|c|}
\hline $\operatorname{PDM} i$ & Participation $\xi_{i}$ & $\begin{array}{c}\text { Correlation } \\
\text { Coefficient } r_{29, j}\end{array}$ & Weight $\lambda_{29, j}$ & $\begin{array}{c}\text { Residual } \\
\text { Participation } \xi_{i}\end{array}$ & $\begin{array}{c}\text { Correlation } \\
\text { Coefficient } r_{13, j}\end{array}$ & Weight $\lambda_{13, j}$ & $\begin{array}{c}\text { Residual } \\
\text { Participation } \xi_{i}\end{array}$ \\
\hline 29 & 0.0453 & 1 & 1 & 0 & 1 & 1 & 1 \\
\hline 34 & 0.0147 & 0.9997 & 0.3270 & 0.0004 & / & / & / \\
\hline 37 & 0.0291 & 0.9959 & 0.6377 & 0.0059 & -0.9935 & -2.274 & 0.0016 \\
\hline 39 & 0.0291 & -0.9959 & -0.6377 & 0.0059 & 0.9935 & 2.274 & 0.0016 \\
\hline 41 & 0.1547 & 0.9995 & 3.393 & 0.0002 & / & / & I \\
\hline 13 & 0.0024 & / & / & 0.0024 & 1 & 1 & 0 \\
\hline 14 & 0.0024 & I & I & 0.0024 & -1.000 & -1.000 & 0 \\
\hline 17 & 0.0026 & / & I & 0.0026 & -1.000 & -1.000 & 0 \\
\hline 18 & 0.0026 & I & I & 0.0026 & 1.000 & 1.000 & 0 \\
\hline 33 & 0.0010 & / & / & 0.0010 & -0.9062 & -0.4038 & 0.0002 \\
\hline
\end{tabular}
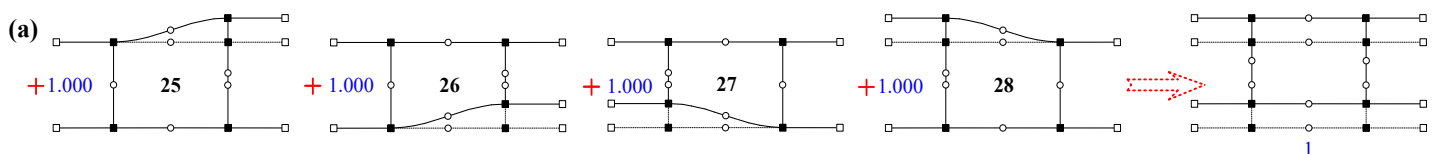

(b)
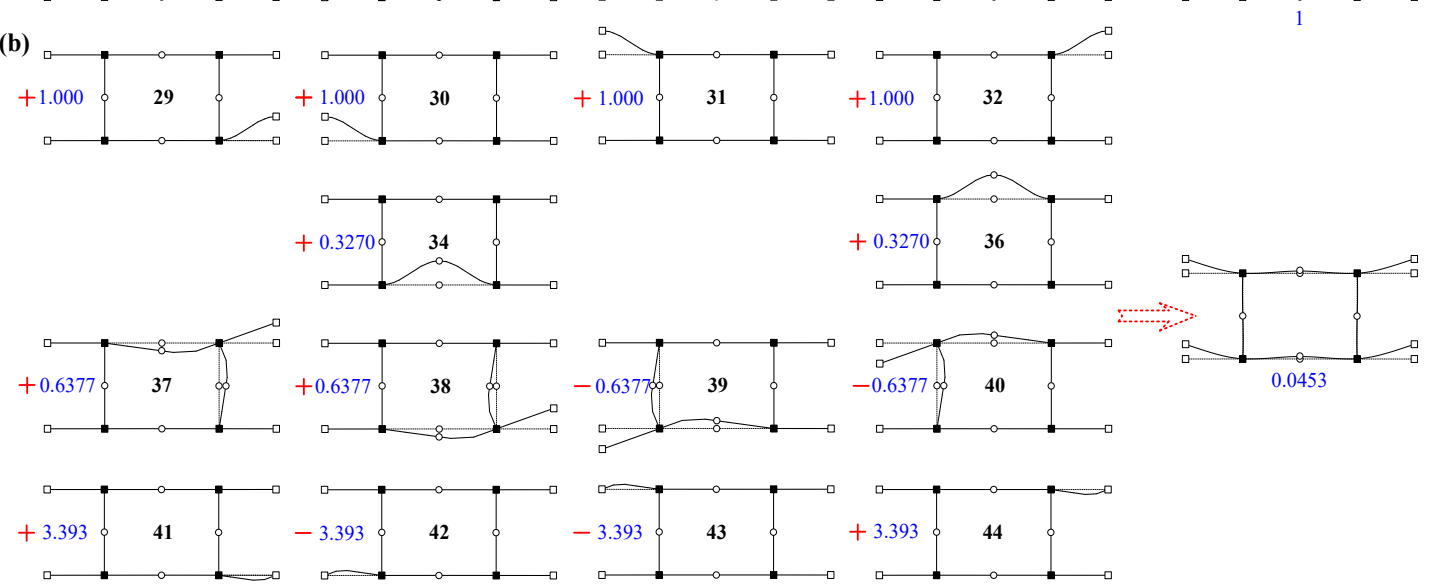

Figure 5. The assembling of in-plane preliminary deformation modes for the first eigenvector of the illustrative thin-walled structure: (a) A classic mode and (b) a distortion mode.

It can be seen that the residual participations of $\chi_{37}{ }^{(1)}$ and $\chi_{39}{ }^{(1)}$ still exceeded 0.001 but was not used for further analysis. This was because the residual component was too weak to provide the information of a structural behaviour. In practice, up to three new deformation modes can be identified from an eigen matrix. Actually, deformation modes related to the residual components are deemed to play more important roles in other eigenvectors with higher orders.

Notice that the amplitude function vectors of Dominant family may not lead to classic mode, but instead may lead to Primary mode in some eigenvectors. For example, the correlation coefficients were $-\gamma_{1,2}=\gamma_{1,3}=-\gamma_{1,4}=1$ in the third eigen matrix, which indicated a primary warping mode. In some cases, the participations of the Dominant family may be lower than the threshold 0.001. For example, there were no EAFV among the Dominant family within the fourth eigen matrix. Then, the in-plane PDM were divided into teams to assemble distortion modes, separately. Moreover, it can be seen that mutual correlation coefficients of amplitude functions belonging to the Dominant family were not 1 or -1 , so information from the two classic modes (or one classic and one primary mode) 
participated in the eigen matrix, that is, the scenarios of DAFC 2. For example, in handling the third eigen matrix, the correlation coefficients were calculated as $\gamma_{25,13}=-\gamma_{25,14}=-\gamma_{25,15}=\gamma_{25,16}=0.9908$ and $\gamma_{25,26}=-\gamma_{25,27}=-\gamma_{25,28}=1$. The results support $\chi_{13}{ }^{(3)}=-\chi_{14}{ }^{(3)}=-\chi_{15}{ }^{(3)}=\chi_{16}{ }^{(3)}$ and $\chi_{25}{ }^{(3)}=$ $\chi_{26}{ }^{(3)}=-\chi_{27}{ }^{(3)}=-\chi_{28}{ }^{(3)}$. Therefore, only $\chi_{13}{ }^{(3)}$ and $\chi_{26}{ }^{(3)}$ were handled, and the results were applicable to the other PDM. According to the signs of the benchmark points, the classic mode was involved in the rotation of about $z$. Thus, in Equation (19), the coefficient can be determined as $\mu_{1}=-4 / 3$. Applying DAFC 2 , both $\chi_{13}{ }^{(3)}$ and $\chi_{26}{ }^{(3)}$ were decomposed into two components, respectively. Figure 6 shows the results, where the components $\chi_{13, I}$ and $\chi_{25, I}$ represent the longitudinal variation of the rotation mode, while $\chi_{13, I I}$ and $\chi_{25, \text { II }}$ correspond to a primary modes. Thus, all the other EAFV were decomposed into two components using DAFC 2, and their weights were calculated through AWDM.
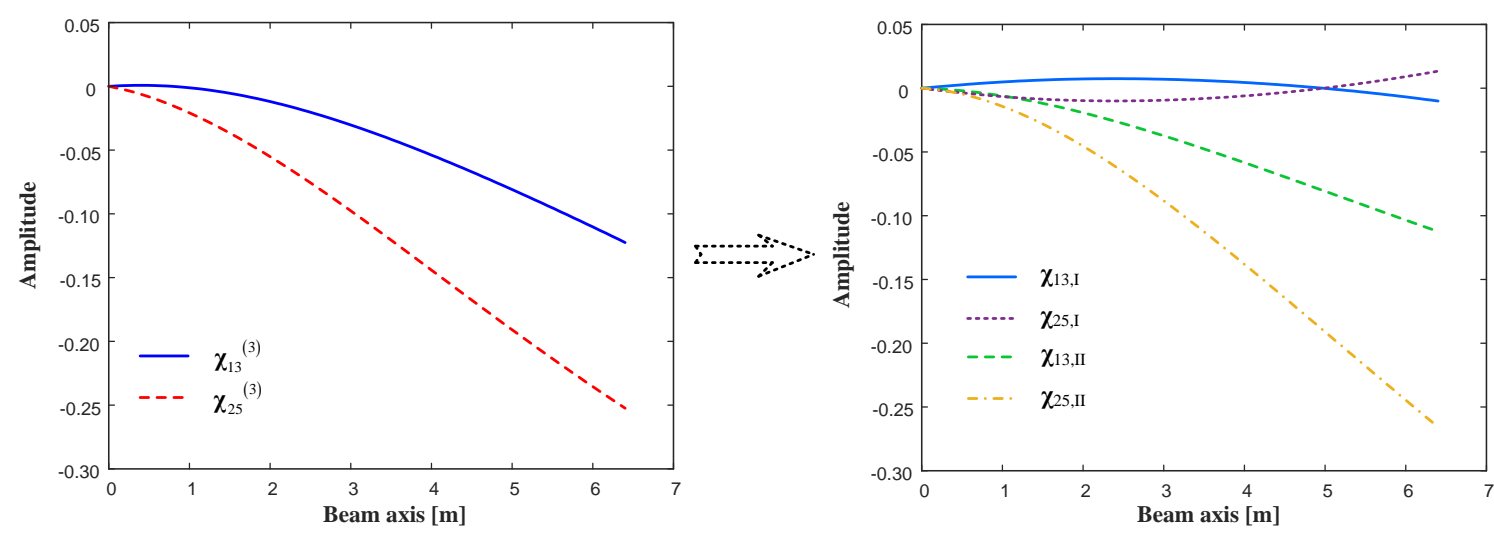

Figure 6. The decomposition result of the second case of decomposing an amplitude function into components (DAFC 2) concerning the third eigen matrix of the illustrative thin-walled structure.

The above procedure was carried out on the first several eigen matrices one by one until the number of identified deformation modes satisfied the requirements. Obviously, the number of the identified deformation modes is unlikely to exceed the number of PDM. Besides, the procedure can be implemented with the help of a computer in a more efficient way. Because the deformation modes are assembled in the light of eigenvectors, their amplitudes should be normalized after the identification. The normalized criteria were simply expressed as rendering the maximum value of each deformation mode to be 1 .

\subsubsection{Numbering of Identified Deformation Modes}

In the previous section, PDM were assembled to provide cross-section deformation modes, which have clear structural interpretation since they stem from the eigenvectors of the governing equations. Moreover, the order of eigenvectors and the participation of PDM reflect the priority of the identified deformation modes, meaning it is possible to organize the deformation modes in hierarchy. Therefore, it is conducive to a reduced higher order beam model.

To this end, it is essential to develop a numbering system that orders the identified deformation modes taking into account of their priority. Generally, the numbering system demands a form filled with all identified deformation modes in the following way: The ones originating from the same eigenvector occupy the same column and the sequence of each column is consistent with the order of the corresponding eigenvector. Within each column, the Classic family is placed on the first row, while the Primary and Secondary families follow them. The numbering is carried out row by row, avoiding double counting of the same deformation mode. Figure 7 shows the numbering system for the illustrative thin-walled structure, where seven out-of-plane modes (modes $i$ to vii) and eleven in-plane modes (modes I to XI) are ordered. 


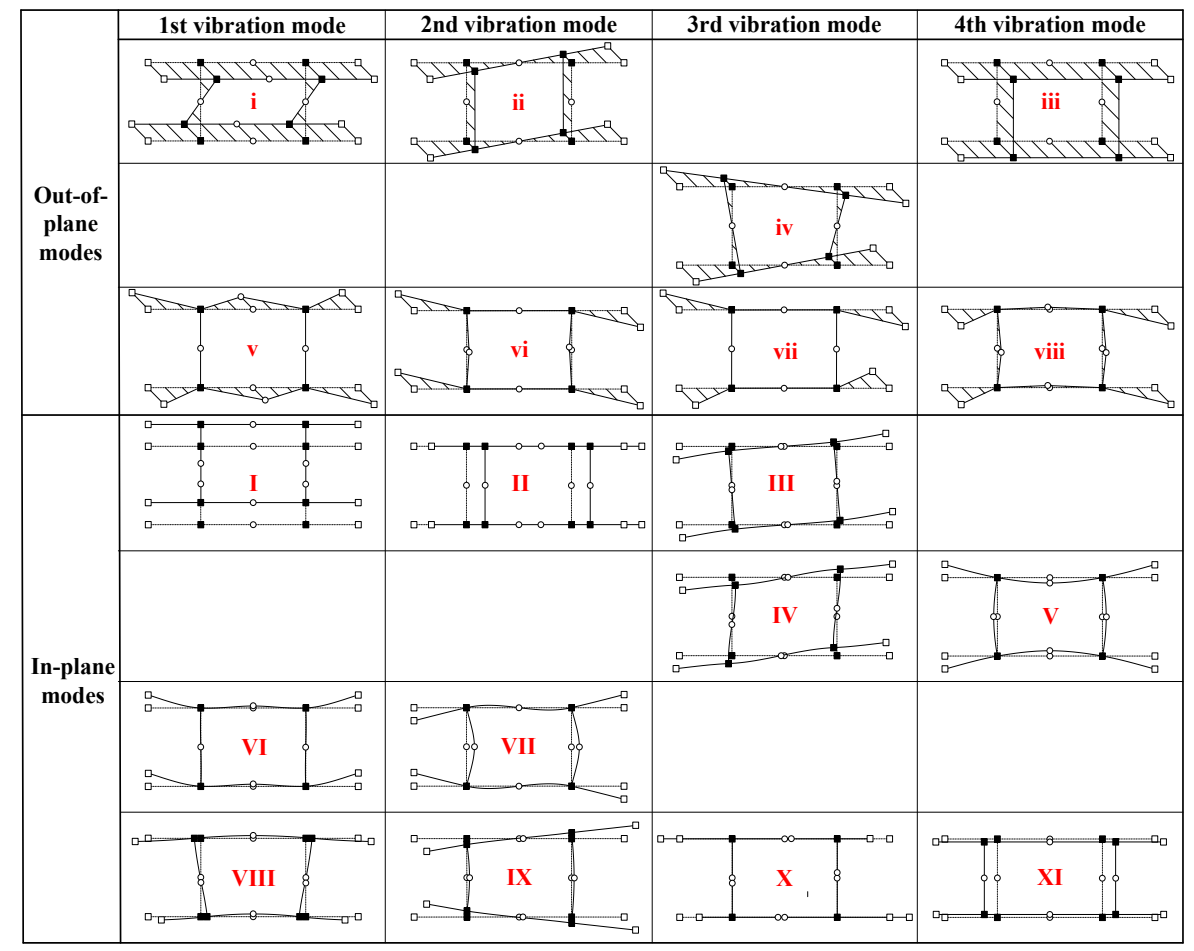

Figure 7. The numbering of deformation modes identified for the illustrative thin-walled structure.

The proposed approach, although involved with cross-section discretization and shape function interpolation, is different from both the theory put forward in [36] to obtain final deformation modes through GBT cross-section analysis and the model developed in [38] to derive a set of deformation modes representing higher order effects applying the uncoupling procedure. In fact, the authors worked on avoiding both the complex subdivision of deformation modes and the solution of nonlinear eigenvalue problem in identifying higher order modes. Instead, a procedure was developed by uncoupling PDM in modal analysis first and then assembling them for cross-section deformation modes considering their participations in eigenvectors. The process lowers the requirements on users, and the deformation modes naturally possess the features of physical interpretation and hierarchy, which is conducive to develop a reduced model.

\section{Illustrative Examples}

The deformation modes identified were divided into three major families considering the hierarchy. New beam models can be developed by employing a selective number of the deformation modes. In order to illustrate the versatility, additional numerical examples were presented concerning some typical cross-sections.

\subsection{Cross-Section Analysis}

In this section, the proposed approach was verified regarding the ability to provide a rational set of deformation modes. The task was carried out on an I-shaped cross-section and a single-cell rectangular cross-section, with the results being compared with those of GBT and another higher order theory, respectively.

\subsubsection{An I-Shaped Cross-Section}

An I-shaped cross-section with material parameters of $E=200 \mathrm{GPa}, v=0.3$ and $\rho=7850 \mathrm{~kg} / \mathrm{m}^{3}$ was considered to exhibit the identification of cross-section deformation modes. The material was linear, elastic and isotropic. This example also shed light on the versatility of the proposed approach in 
coping with open cross-sections. The cross-section had a web with dimensions $80 \mathrm{~mm} \times 2 \mathrm{~mm}$ and two flanges with dimensions $75 \mathrm{~mm} \times 2 \mathrm{~mm}$. A cantilevered beam, with cross-section and a length $L=$ $450 \mathrm{~mm}$, was modelled using the proposed theory. The cross-section discretization was performed with six natural nodes and one artificial node on the middle point of the web. Figure 8 displays the output of the identification by handling the first four eigenvectors of the thin-walled structure using the proposed approach. These deformation modes were classified into three families: Modes i, ii, iii, I, II and III belonged to the Classic family, while modes iv and IV belonged to the Primary family and the others belonged to the Secondary family.
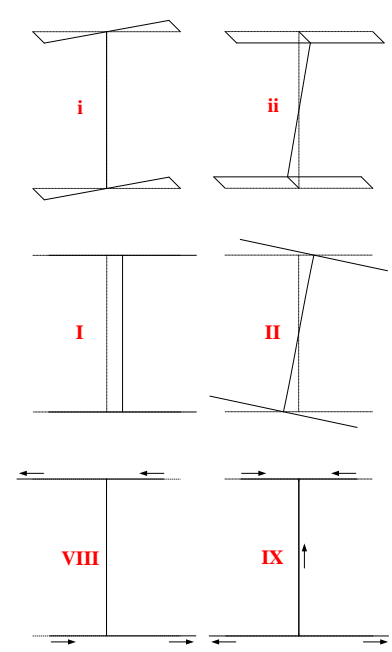
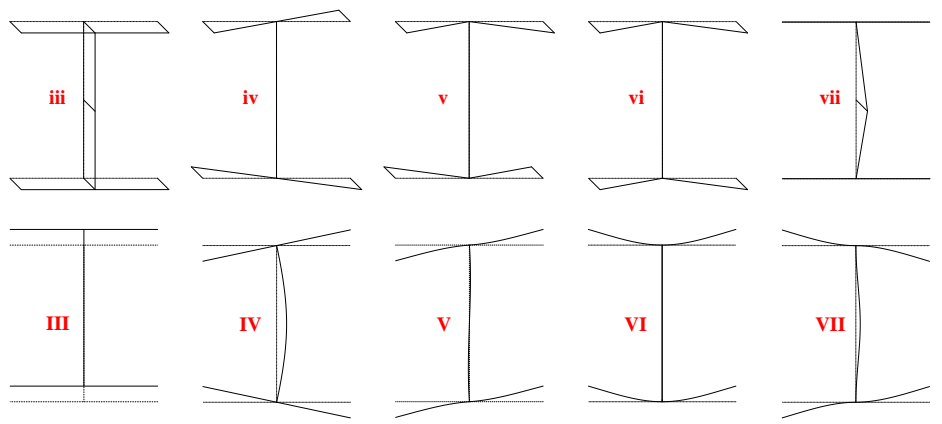

Figure 8. The cross-section deformation modes identified from the first four eigenvectors of the illustrative thin-walled structure applying the proposed approach.

The results were compared with the first 20 GBT deformation modes [38]. As shown in Figure 9, the proposed approach captured most of the GBT modes by dealing with just the first four eigenvectors of the thin-walled structure. Actually, these cross-section deformation modes are qualified to reproduce the 3D deformation of the first 10 vibration modes of the thin-walled structure, which covered most engineering cases. When the other deformation modes with lower priority are needed, all one needs to do is handle more eigenvectors. The quality is conductive to obtain a selective set of deformation modes according to the actual needs of the structural analysis, instead of calculating all of them from the very beginning. Thus, the proposed approach is convenient and efficient in adapting different demands.
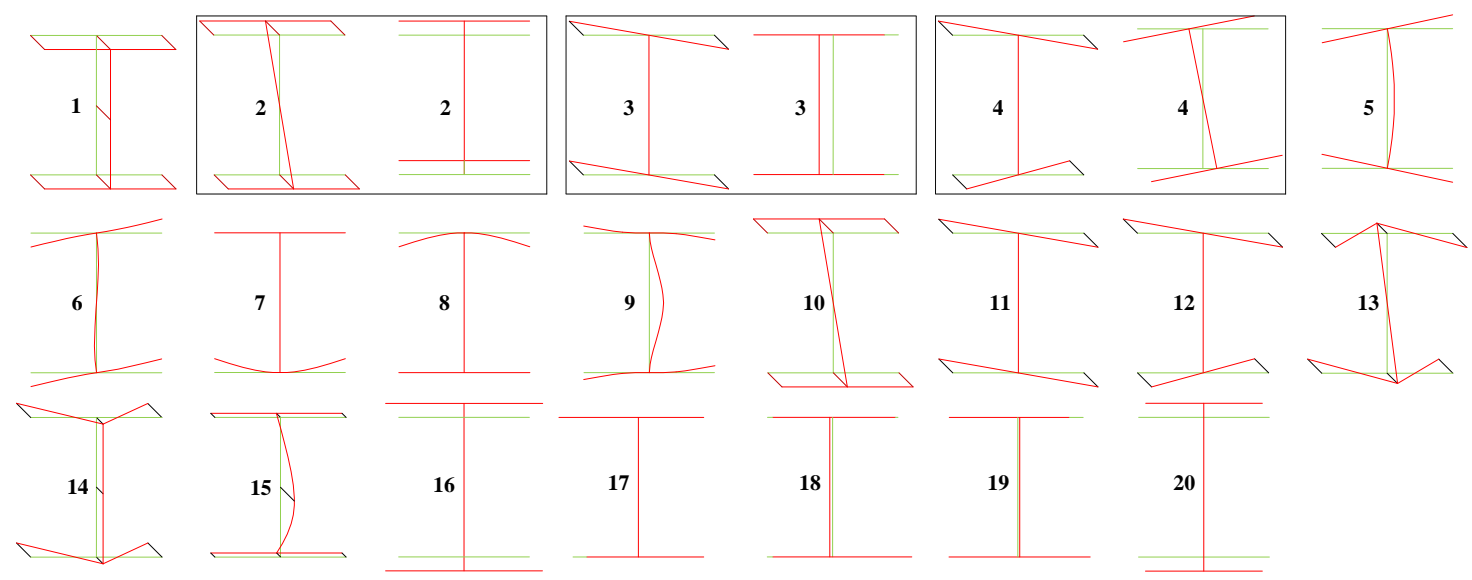

Figure 9. The generalized beam theory (GBT) deformation modes of the illustrative thin-walled structure derived with GBTUL 2.0. 
There were also some differences between the two sets of cross-section deformation modes. For example, the in-plane and out-of-plane modes were tied together to describe the structural behaviours related to flexure and rotation in GBT, while they were identified separately in the proposed approach. However, the difference will not influence the accuracy of the thin-walled model, since the modes 2, 3, 4, 10, 11 and 12 tied together in Figure 9 were equal to the linear combination of modes i, ii, iv, I, II and III, respectively. Besides, the pattern to exhibit the structural interpretation of deformation modes was not quite the same. For example, modes 7 and 8 were separately given in Figure 9, while the two were assembled with weights 1 and -1 in Figure 8. It is predictable that another mode assembled with weights 1 and 1 can be identified by handling higher order eigenvectors. This difference was independent of the model accuracy, but the modes shown in Figure 8 were closer to the actual structural behaviours.

Overall, the proposed approach can capture the cross-section deformation modes of an open cross-section with a convenient and efficient way. The derived deformation modes were generally consistent with GBT, though there were some differences that independent of the model accuracy.

\subsubsection{A Single-Cell Rectangular Cross-Section}

The thin-walled structure with a single-cell rectangular cross-section was analysed by the proposed approach to highlight its capability in coping with closed cross-sections. The cross-section was made of concrete, which was linear, elastic and isotropic, with the material parameters $E=21 \mathrm{GPa}, v=0.2$ and $\rho=2500 \mathrm{~kg} / \mathrm{m}^{3}$. The cross-section had a width of $b=2 \mathrm{~m}$, a height of $h=1 \mathrm{~m}$ and a constant thickness of $t=0.15 \mathrm{~m}$. A cantilevered beam, having the cross-section and a length $L=15 \mathrm{~m}$, was handled using the proposed approach to obtain the deformation modes, where the cross-section discretization was performed with five nodes on each width and three nodes on each height.

The warping modes for the single-cell rectangular cross-section are displayed in Figure 10. These modes were derived from the first six eigenvectors of the thin-walled structure using the proposed approach. More concretely, modes v, vi and ix were derived from the first three eigenvectors (associated with the flexure), while modes iv and vii both were derived from the fourth eigenvector (associated with the rotation) and mode viii was related to the fifth eigenvector (associated with the uniform extension). From the point of hierarchy, mode iv belonged to the Primary family, while the others belonged to the Secondary family.
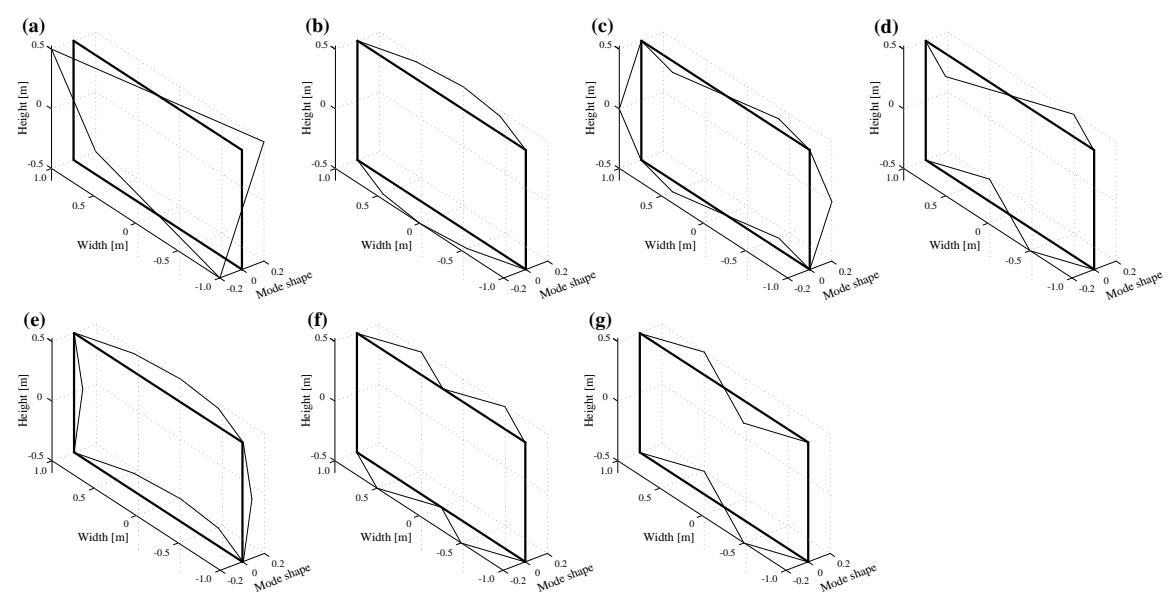

Figure 10. The first seven warping modes of the single-cell rectangular cross-section derived with the proposed approach: (a) Mode iv, (b) mode v, (c) mode vi, (d) mode vii, (e) mode viii, (f) mode ix and (g) mode $x$.

Figure 11 presents the warping modes for the cross-section obtained by Vieira et al. [37]. The cross-section discretization was carried out in the same way. The modes were organized 
hierarchically, where the first two represent shear-lag modes associated with the flexure modes, and the other two represent shear-lag modes associated with the extension and shear deformation, respectively. By comparison, the four deformation modes in Figure 11 correspond to modes vi, v, viii and ix, respectively. The difference occurred because the deformation modes in Figure 11 still contained the components of Classic modes, while those in Figure 10 eliminated these products. For example, the first mode in Figure 11 can be viewed as the combination of mode vi and Classic mode ii (flexure about the major axis) in the system of the proposed model. The same style goes for the remaining three modes. Thus, the two approaches were in well-agreement in the sense that all the warping modes obtained through the uncoupling procedure can be captured with the set of out-of-plane deformation modes derived with the proposed approach, although the priority assignments of two systems were not fully consistent. However, Primary warping mode iv (Vlasov warping) was not represented explicitly with the uncoupling procedure in [37], which goes against with mainstream theories.
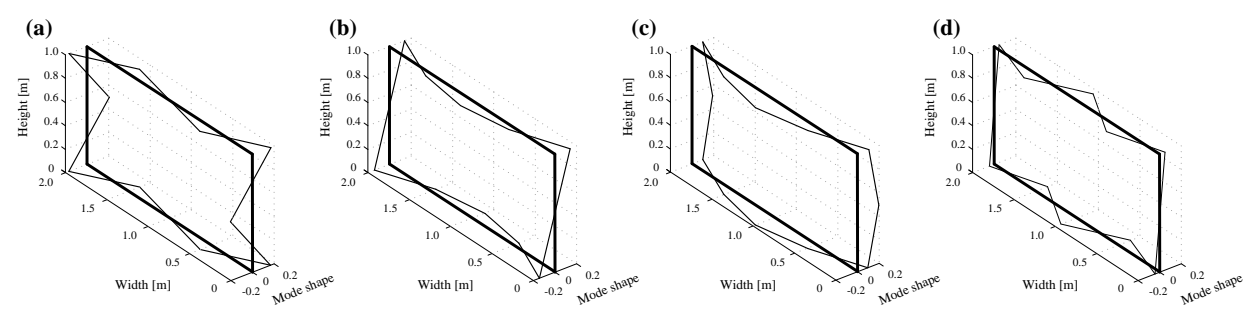

Figure 11. The first four warping modes of the single-cell rectangular cross-section derived by Vieira et al. [37]: (a) Shear-lag mode 1, (b) shear-lag mode 2, (c) shear-lag mode 3 and (d) a higher order mode.

\subsection{Beam Analysis}

A cantilevered thin-walled beam with the illustrative cross-section shown in Figure 2 and a beam length of $L=6.4 \mathrm{~m}$ was considered for dynamic analyses in order to verify the performance of the proposed higher order models. The beam was meshed with 40 proposed quadratic elements equally distributed along the beam axis. Besides, a 3D shell model considering a mesh of $44 \times 64$ Shell 181 elements over the cross-section and along the beam axis, respectively, was implemented in ANSYS 17.0 in order to compare with the results of the proposed models. Here, the material was assumed to be linear, elastic and isotropic in the calculation, and the beam was under the small displacement hypothesis in the whole vibration process.

\subsubsection{Concerning the Accuracy and Efficiency}

In order to verify the accuracy and efficiency of the proposed models, comparisons regarding the natural frequencies were carried out with ANSYS shell theory. Selectively, several sets of cross-section deformation modes were constructed considering the hierarchy and used to derive the corresponding higher order models. One set includes all the 48 PDM and yielded the proposed beam 1; another set has six Classic modes and results in the classic beam; the third set has six Classic modes and three Primary modes, corresponding to the proposed beam 2; the fourth set has all the modes of set 3 and two Secondary modes VI and VII included, reaching the proposed beam 3; the last set added three Secondary modes $\mathrm{v}$, vi and vii on the basis of set 4 , and corresponds to the proposed beam 4 . Table 3 shows the results, where the relative errors are calculated based on the assumption that the values derived with ANSYS shell model are exact enough.

Table 3 shows a clear trend of gradual improvement on the accuracy regarding the natural frequencies as the number of employed cross-section deformation modes increases. It is also noticeable that the proposed beam model applying 14 cross-section deformation modes had a good agreement with the numerical results obtained from ANSYS shell model regarding the first 10 natural frequencies, while the classic beam model was not competent to perform the dynamic analysis, even for the first 
two natural frequencies. Meanwhile, the results support that it is essential to consider cross-section deformation modes in the analysis of thin-walled structures. It can also be seen that the set of deformation modes, including only Classic and Primary modes, were able to form a higher order model to catch the first eight natural frequencies (the sixth not included) of a thin-walled structure with relative errors lower than $4.90 \%$. The ability can be improved to calculate the first 10 natural frequencies with relative errors lower than $4.15 \%$ when eight Secondary modes are added to the proposed beam model. These results specify the accuracy of the proposed model, as well as the rational hierarchy of the proposed numbering system.

Table 3. Comparison of the first 10 natural frequencies of the illustrative thin-walled structure modelled with the proposed theory and ANSYS Shell 181, respectively.

\begin{tabular}{|c|c|c|c|c|c|c|c|c|c|c|c|}
\hline \multirow{2}{*}{$\begin{array}{c}\text { Mode } \\
\text { Number }\end{array}$} & \multirow{2}{*}{$\begin{array}{c}\text { ANSYS } \\
\text { Shell } \\
\begin{array}{c}\text { Frequency } \\
{[\mathrm{Hz}]}\end{array}\end{array}$} & \multicolumn{2}{|c|}{ Proposed Beam 1} & \multicolumn{2}{|c|}{ Classic Beam } & \multicolumn{2}{|c|}{ Proposed Beam 2} & \multicolumn{2}{|c|}{ Proposed Beam 3} & \multicolumn{2}{|c|}{ Proposed Beam 4} \\
\hline & & $\begin{array}{c}\text { Frequency } \\
{[\mathrm{Hz}]}\end{array}$ & $\begin{array}{l}\text { Relative } \\
\text { Error [\%] }\end{array}$ & $\begin{array}{c}\text { Frequency } \\
{[\mathrm{Hz}]}\end{array}$ & $\begin{array}{c}\text { Relative } \\
\text { Error [\%] }\end{array}$ & $\begin{array}{c}\text { Frequency } \\
{[\mathrm{Hz}]}\end{array}$ & $\begin{array}{c}\text { Relative } \\
\text { Error [\%] }\end{array}$ & $\begin{array}{c}\text { Frequency } \\
{[\mathrm{Hz}]}\end{array}$ & $\begin{array}{l}\text { Relative } \\
\text { Error [\%] }\end{array}$ & $\begin{array}{c}\text { Frequency } \\
{[\mathrm{Hz}]}\end{array}$ & $\begin{array}{c}\text { Relative } \\
\text { Error [\%] }\end{array}$ \\
\hline 1 & 17.83 & 17.91 & 0.45 & 18.77 & 5.27 & 18.66 & 4.65 & 18.57 & 4.15 & 18.48 & 3.64 \\
\hline 2 & 29.01 & 29.16 & 0.52 & 30.63 & 5.58 & 30.43 & 4.89 & 30.35 & 4.62 & 30.16 & 3.96 \\
\hline 3 & 43.20 & 42.56 & -1.48 & 77.52 & 79.4 & 44.11 & 2.11 & 44.11 & 2.11 & 43.20 & 0 \\
\hline 4 & 57.73 & 56.97 & -1.32 & / & / & 57.11 & -1.07 & 57.11 & -1.07 & 57.11 & -1.07 \\
\hline 5 & 60.56 & 58.86 & -2.81 & l & l & 59.89 & -1.11 & 59.89 & -1.11 & 59.89 & -1.11 \\
\hline 6 & 65.10 & 63.74 & -2.09 & / & / & / & / & I & / & 64.23 & -1.34 \\
\hline 7 & 66.62 & 64.69 & -2.90 & / & / & 65.13 & -2.24 & 65.13 & -2.24 & 65.13 & -2.24 \\
\hline 8 & 72.53 & 72.01 & -0.72 & I & l & 72.30 & -0.32 & 72.30 & -0.32 & 72.30 & -0.32 \\
\hline 9 & 75.31 & 76.27 & 1.27 & I & I & I & I & 78.34 & 4.02 & 78.20 & 3.84 \\
\hline 10 & 75.71 & 76.95 & 1.64 & I & I & I & I & 78.84 & 4.13 & 78.84 & 4.13 \\
\hline
\end{tabular}

One can also have an insight of the hierarchy of the identified cross-section deformation modes through their participation in the eigenvectors of the thin-walled structure. Figure 12 shows their amplitude functions varying along the beam axis concerning the first four eigenvectors. A clear distinction can be observed among the participations of Classic, Primary and Secondary modes. Generally, the participation of Classic mode is dozens of times of that of Primary mode, and so is that of Primary mode and Secondary mode. Just because of this character, a rational hierarchy becomes possible in organizing identified cross-section deformation modes. And because of the hierarchy, one is able to select several cross-section deformation modes with high priority to develop a reduced beam model based on actual demands. This effectively contributes to the improvement of efficiency in computation, since fewer degrees of freedom should be dealt with in the thin-walled model without a significant loss of accuracy.
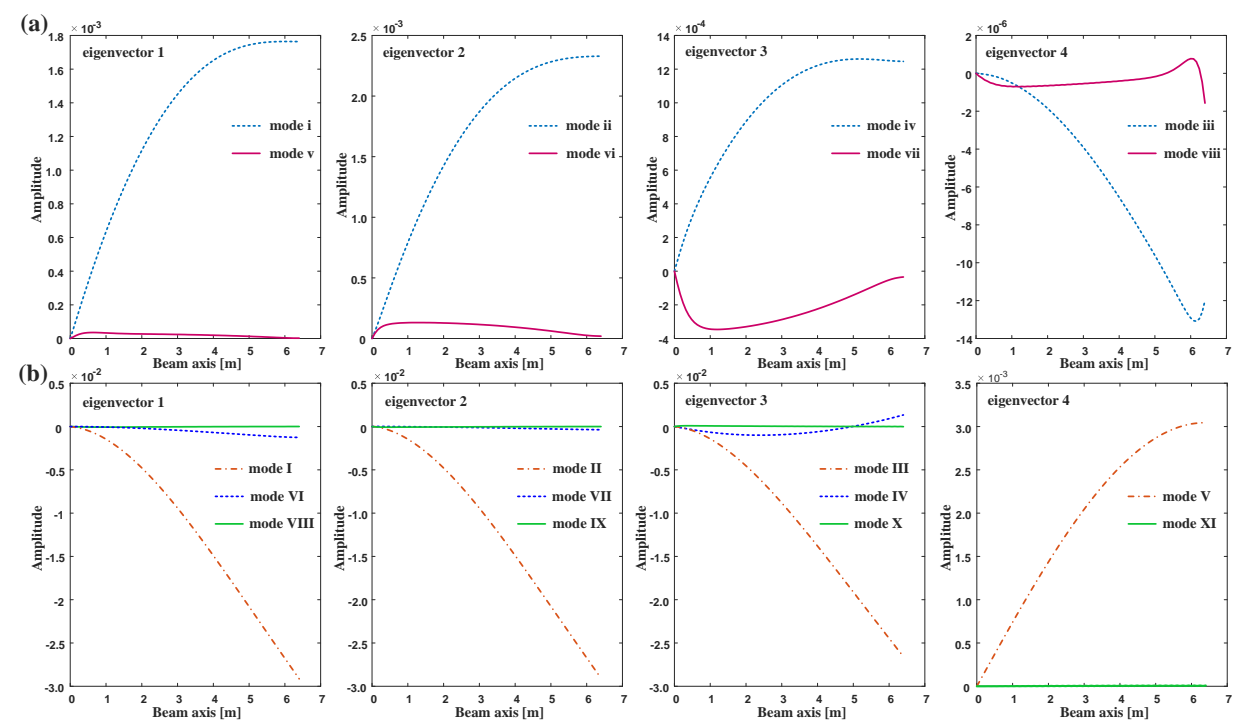

Figure 12. The amplitude functions of the identified cross-section deformation modes varying along the beam axis concerning the first four eigenvectors: (a) Out-of-plane modes and (b) in-plane modes. 
Moreover, one should bear in mind that the results of the proposed models were obtained with a mesh of 40 1D elements, while those in the comparison were derived with a 3D shell model adopting 2816 2D shell elements, notably having a comparable accuracy. This was a great improvement of the computation efficiency in the analysis of thin-walled structures.

\subsubsection{Concerning the Reproduction of 3D Deformation}

The vibration modes of the illustrative thin-walled structure were presented in order to exhibit the applicability of the proposed model in performing the 3D analysis of thin-walled structures. The results obtained were compared with finite element models implemented in ANSYS 17.0. Toward the plotting of the deformed configuration, the displacement field obtained from the proposed model was computed in a "mesh" of sampling points. The mesh was defined over the beam cross-section with 44 points and lengthwise along the beam axis with 64 points. Thus, $44 \times 64$ squares were employed to describe the thin-walled structure configuration. To this end, the displacements of these sampling points were obtained from the finite element solution of the proposed beam model and then used to compute the new coordinates of these sampling points. Then, in a global coordinate system describing the deformed configuration, these sampling points were interconnected over the cross-section and along the beam axis, respectively. It should be noted that the mesh was just used to represent the deformed configuration and not the discretization adopted for the cross-section analysis or the finite element solution.

Figure 13 shows the comparison regarding the third to tenth vibration modes of the illustrative thin-walled structure. The first and second vibration modes were not listed because the cross-section deformation was not obvious enough and thus may not be typical in exhibiting the capability of reproducing three-dimensional deformation. The comparison was divided into eight pairs according to the order of the vibration modes. Within each pair, the left was derived with the proposed beam 4 , while the right represents the ANSYS shell results. In general, there was no visible difference between all the eight pairs. This proved the capability of the proposed model in predicting the vibration modes of thin-walled structures. Simultaneously, the comparison reconfirmed the good agreements between the proposed model and ANSYS shell model. In this sense, it is rational to believe that the proposed model can accurately reproduce 3D deformation and forecast the dynamic behaviours of thin-walled structures.

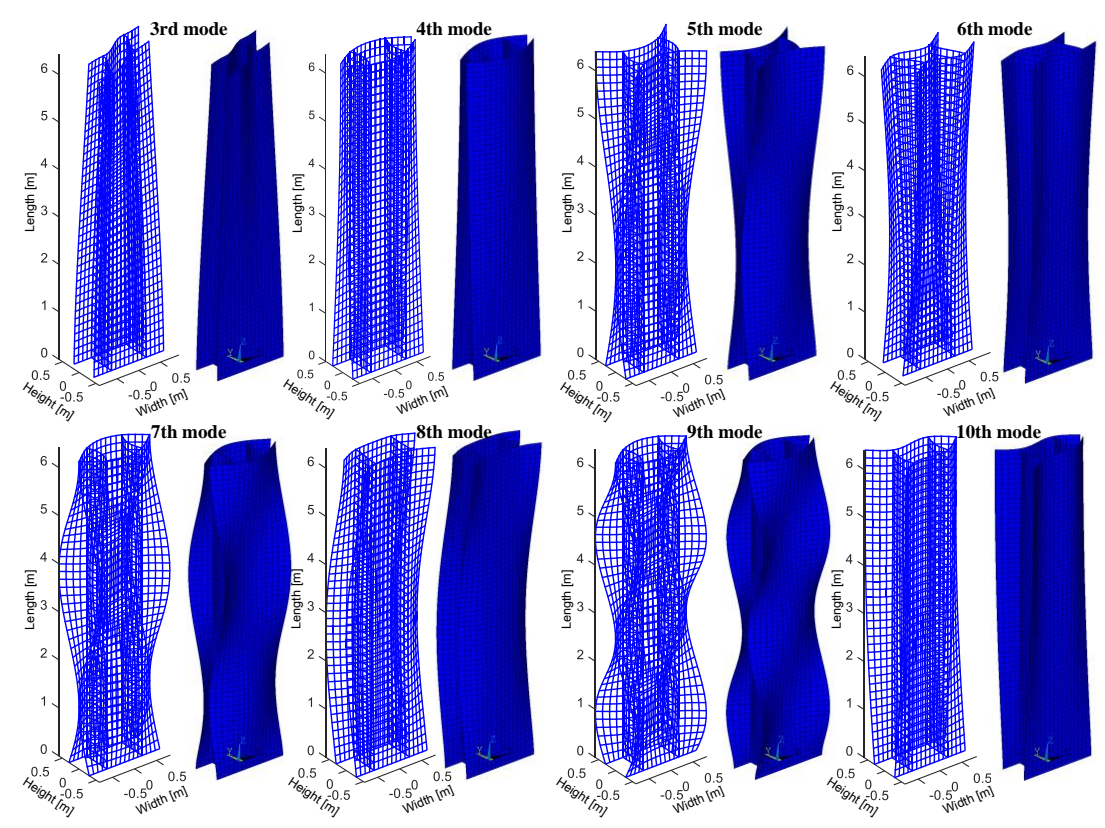

Figure 13. The comparison between the proposed model and ANSYS shell model concerning the third to tenth vibration modes of the illustrative thin-walled structure. 


\section{Conclusions}

This paper presented and discussed the main concepts and operations involved in the identification of cross-section deformation modes through the assembling of PDM in the framework of a higher order beam theory. The process focused on and settled three key issues, including (i) the determination of PDM that can be assembled together, (ii) the assignation of the corresponding weights that indicate their participation and (iii) the decomposition of an amplitude function when related to different structural behaviours. The assembled deformation modes were organized considering the hierarchy and structural characteristics, and selectively used to update the governing equations of reduced higher order models. The main features of this novel approach are found in its ability to be numerically implemented in a more manageable way and the resulted set of cross-section deformation modes being hierarchically organized and of clear mechanical interpretation, which are conducive to develop a reduced model without a significant loss of accuracy. These properties were proved through numerical examples concerning some typical cross-sections and comparison with other theories. Next, the authors plan to develop the approach to handle cross-sections with curved walls.

Author Contributions: Conceptualization, W.Z.; Methodology, L.Z.; software, A.J.; Writing—original draft preparation, L.Z.; Writing-review and editing, W.Z.; Funding acquisition, A.J.

Funding: This research was funded by the National Natural Science Foundation of China (Grant Nos. 51805144 and 11772100), the Natural Science Foundation of Jiangsu Province (Grant No. BK20170300), and the Changzhou Sci\&Tech Program (Grant No. CZ20190018).

Conflicts of Interest: The authors declare no conflict of interest.

\section{References}

1. Yang, Z.; Xu, C. Research on Compression Behavior of Square Thin-Walled CFST Columns with Steel-Bar Stiffeners. Appl. Sci. 2018, 8, 1602. [CrossRef]

2. El Fatmi, R. A refined 1D beam theory built on 3D Saint-Venant's solution to compute homogeneous and composite beams. J. Mech. Mater. Struct. 2016, 11, 345-378. [CrossRef]

3. Genoese, A.; Genoese, A.; Bilotta, A.; Garcea, G. A geometrically exact beam model with non-uniform warping coherently derived from the Saint Venant rod. Eng. Struct. 2014, 68, 33-46. [CrossRef]

4. Naccache, F.; El Fatmi, R. Buckling analysis of homogeneous or composite I-beams using a 1D refined beam theory built on Saint Venant's solution. Thin Walled Struct. 2018, 127, 822-831. [CrossRef]

5. Naccache, F.; El Fatmi, R. Numerical free vibration analysis of homogeneous or composite beam using a refined beam theory built on Saint Venant's solution. Comput. Struct. 2018, 210, 102-121. [CrossRef]

6. Carrera, E.; Pagani, A.; Petrolo, M.; Zappino, E. Recent developments on refined theories for beams with applications. Mech. Eng. Rev. 2015, 2. [CrossRef]

7. Berdichevskii, V. Variational-asymptotic method of constructing a theory of shells. J. Appl. Math. Mech. 1979, 43, 711-736. [CrossRef]

8. Zhang, L.; Sertse, H.M.; Yu, W. Variational asymptotic homogenization of finitely deformed heterogeneous elastomers. Compos. Struct. 2019, 216, 379-391. [CrossRef]

9. Harursampath, D.; Harish, A.B.; Hodges, D.H. Model reduction in thin-walled open-section composite beams using variational asymptotic method. Part I: Theory. Thin Walled Struct. 2017, 117, 356-366. [CrossRef]

10. Jiang, F.; Deo, A.; Yu, W. A composite beam theory for modeling nonlinear shear behavior. Eng. Struct. 2018, 155, 73-90. [CrossRef]

11. Carrera, E.; Petrolo, M. On the effectiveness of higher-order terms in refined beam theories. J. Appl. Mech. 2011, 78, 021013. [CrossRef]

12. Pagani, A.; Yan, Y.; Carrera, E. Exact solutions for static analysis of laminated, box and sandwich beams by refined layer-wise theory. Compos. Part B Eng. 2017, 131, 62-75. [CrossRef] 
13. Yan, Y.; Pagani, A.; Carrera, E. Exact solutions for free vibration analysis of laminated, box and sandwich beams by refined layer-wise theory. Compos. Struct. 2017, 175, 28-45. [CrossRef]

14. Carrera, E.; Pagani, A.; Banerjee, J.R. Linearized buckling analysis of isotropic and composite beam-columns by Carrera Unified Formulation and Dynamic Stiffness Method. Mech. Adv. Mater. Struct. 2016, 23, 1092-1103. [CrossRef]

15. Yan, Y.; Carrera, E.; De Miguel, A.; Pagani, A.; Ren, Q.W.; Miguel, A. Meshless analysis of metallic and composite beam structures by advanced hierarchical models with layer-wise capabilities. Compos. Struct. 2018, 200, 380-395. [CrossRef]

16. Vlassov, V. Thin-Walled Elastic Beams; Israel Program for Scientific Translations: Jerusalem, Israel, 1961.

17. Capdevielle, S.; Grange, S.; Dufour, F.; Desprez, C. A multifiber beam model coupling torsional warping and damage for reinforced concrete structures. Eur. J. Environ. Civ. Eng. 2016, 20, 914-935. [CrossRef]

18. Yoon, K.; Lee, P.S.; Kim, D.N. An efficient warping model for elastoplastic torsional analysis of composite beams. Compos. Struct. 2017, 178, 37-49. [CrossRef]

19. Lim, T.C. Refined shear correction factor for very thick simply supported and uniformly loaded isosceles right triangular auxetic plates. Smart Mater. Struct. 2016, 25, 54001. [CrossRef]

20. Pavazza, R.; Blagojevic, B. On the cross-section distortion of thin-walled beams with multi-cell cross-sections subjected to bending. Int. J. Solids Struct. 2005, 42, 901-925. [CrossRef]

21. Park, N.H.; Choi, S.; Kang, Y.J. Exact distortional behavior and practical distortional analysis of multicell box girders using an expanded method. Comput. Struct. 2005, 83, 1607-1626. [CrossRef]

22. Cambronero-Barrientos, F.; Díaz-Del-Valle, J.; Martínez-Martínez, J.A. Beam element for thin-walled beams with torsion, distortion, and shear lag. Eng. Struct. 2017, 143, 571-588. [CrossRef]

23. Zhang, Y.H.; Lin, L.X. Shear lag analysis of thin-walled box girders based on a new generalized displacement. Eng. Struct. 2014, 61, 73-83. [CrossRef]

24. Schardt, R. Generalized beam theory-An adequate method for coupled stability problems. Thin Walled Struct. 1994, 19, 161-180. [CrossRef]

25. Gonçalves, R.; Camotim, D.; Peres, N. Application of Generalised Beam Theory to curved members with circular axis. Stahlbau 2018, 87, 345-354. [CrossRef]

26. Gonçalves, R.; Camotim, D. On the first-order and buckling behaviour of thin walled regular polygonal tubes. Steel Constr. 2016, 9, 279-290. [CrossRef]

27. Bebiano, R.; Basaglia, C.; Camotim, D.; Gonçalves, R. GBT buckling analysis of generally loaded thin-walled members with arbitrary flat-walled cross-sections. Thin Walled Struct. 2018, 123, 11-24. [CrossRef]

28. Bebiano, R.; Eisenberger, M.; Camotim, D.; Gonçalves, R. GBT-Based Vibration Analysis Using the Exact Element Method. Int. J. Struct. Stab. Dyn. 2018, 18, 1850068. [CrossRef]

29. Ruggerini, A.; Madeo, A.; Gonçalves, R.; Camotim, D.; Ubertini, F.; De Miranda, S. GBT post-buckling analysis based on the Implicit Corotational Method. Int. J. Solids Struct. 2019, 163, 40-60. [CrossRef]

30. Bebiano, R.; Calçada, R.; Camotim, D.; Silvestre, N. Dynamic analysis of high-speed railway bridge decks using generalised beam theory. Thin Walled Struct. 2017, 114, 22-31. [CrossRef]

31. Vieira, R.; Virtuoso, F.; Pereira, E. A higher order beam model for thin-walled structures with in-plane rigid cross-sections. Eng. Struct. 2015, 84, 1-18. [CrossRef]

32. Vieira, R.; Virtuoso, F.; Pereira, E. Buckling of thin-walled structures through a higher order beam model. Comput. Struct. 2017, 180, 104-116. [CrossRef]

33. Vieira, R.; Virtuoso, F.; Pereira, E. Definition of warping modes within the context of a higher order thin-walled beam model. Comput. Struct. 2015, 147, 68-78. [CrossRef]

34. Vieira, R.F.; Virtuoso, F.; Pereira, E.; Virtuoso, F. A higher order model for thin-walled structures with deformable cross-sections. Int. J. Solids Struct. 2014, 51, 575-598. [CrossRef]

35. Zhang, L.; Zhu, W.; Ji, A.; Peng, L. A Simplified Approach to Identify Sectional Deformation Modes of Thin-Walled Beams with Prismatic Cross-Sections. Appl. Sci. 2018, 8, 1847. [CrossRef]

36. Bebiano, R.; Gonçalves, R.; Camotim, D. A cross-section analysis procedure to rationalise and automate the performance of GBT-based structural analyses. Thin Walled Struct. 2015, 92, 29-47. [CrossRef] 
37. Zhang, L.; Ji, A.; Zhu, W.; Peng, L. On the Identification of Sectional Deformation Modes of Thin-Walled Structures with Doubly Symmetric Cross-Sections Based on the Shell-Like Deformation. Symmetry 2018, 10, 759. [CrossRef]

38. Bebiano, R.; Camotim, D.; Gonçalves, R. GBTul 2.0—A second-generation code for the GBT-based buckling and vibration analysis of thin-walled members. Thin Walled Struct. 2018, 124, 235-257. [CrossRef]

(C) 2019 by the authors. Licensee MDPI, Basel, Switzerland. This article is an open access article distributed under the terms and conditions of the Creative Commons Attribution (CC BY) license (http://creativecommons.org/licenses/by/4.0/). 